This item was submitted to Loughborough's Research Repository by the author.

Items in Figshare are protected by copyright, with all rights reserved, unless otherwise indicated.

\title{
Air-related mechanisms of noise generation by solid rubber tyres with cavities
}

PLEASE CITE THE PUBLISHED VERSION

PUBLISHER

(C) Elsevier

VERSION

AM (Accepted Manuscript)

LICENCE

CC BY-NC-ND 4.0

\section{REPOSITORY RECORD}

Eisenblaetter, Jochen, Stephen J. Walsh, and Victor V. Krylov. 2010. "Air-related Mechanisms of Noise Generation by Solid Rubber Tyres with Cavities". figshare. https://hdl.handle.net/2134/6481. 
This item was submitted to Loughborough's Institutional Repository (https://dspace.lboro.ac.uk/) by the author and is made available under the following Creative Commons Licence conditions.

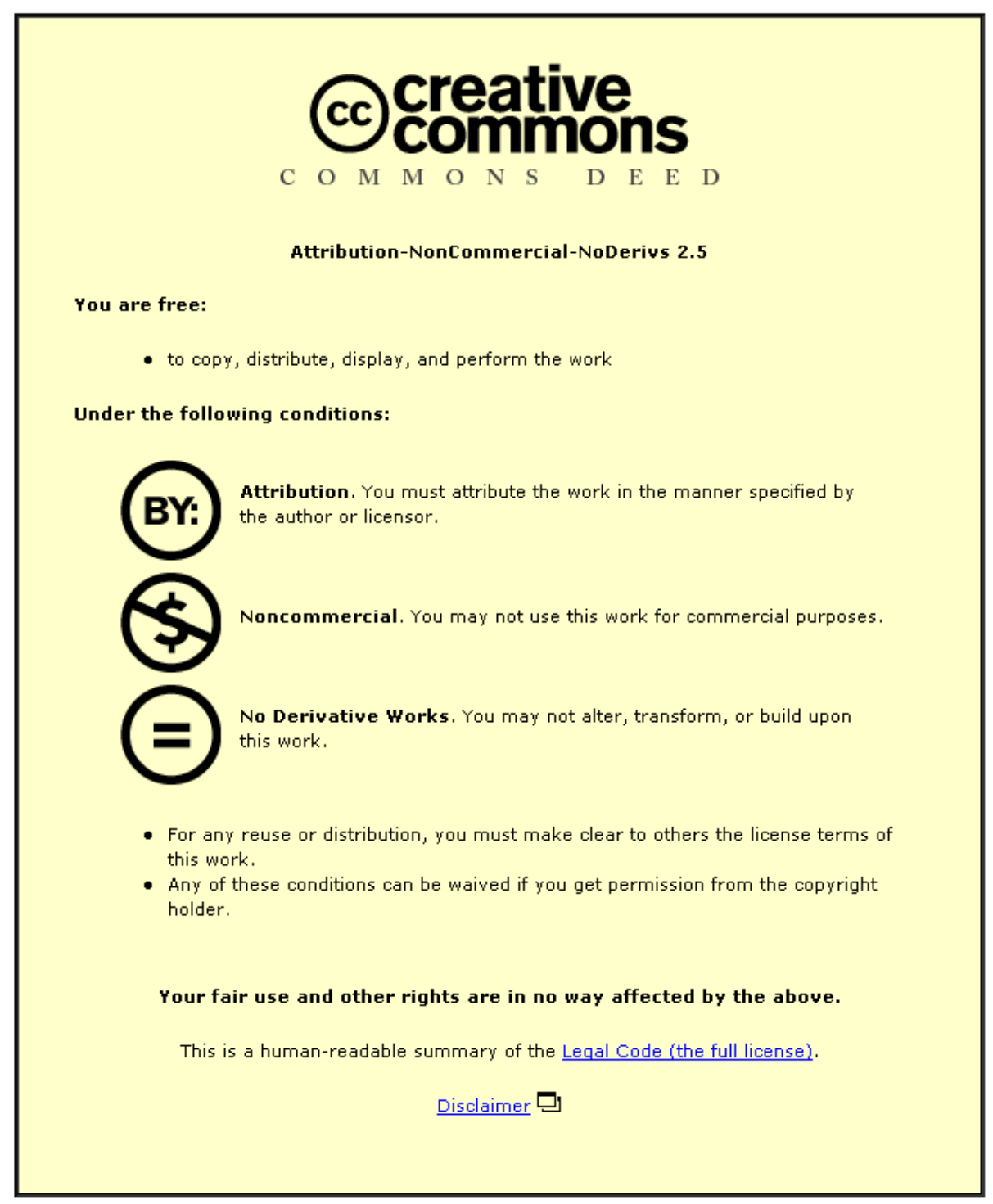

For the full text of this licence, please go to: http://creativecommons.org/licenses/by-nc-nd/2.5/ 


\title{
Air-related mechanisms of noise generation by solid rubber tyres with cavities.
}

Jochen Eisenblaetter, Stephen J. Walsh and Victor V. Krylov

Department of Aeronautical and Automotive Engineering, Loughborough University, Loughborough, Leicestershire, LE11 3TU, UK

Corresponding author's email address: S.J.Walsh@lboro.ac.uk

\begin{abstract}
There are four main air-related noise generation mechanisms at the tyre/road interface, which were all categorised more than 20 years ago. The first one is the so-called 'air pumping' mechanism. Two other air-related phenomena that occur when there are air movements near the contact patch of the tyre are 'air resonant radiation' and 'pipe
\end{abstract}


resonances' which appear at the footprint of the tyre. In addition to these, there is a forth effect, which is mentioned in the literature, that is occurring due to turbulence effects of the air surrounding the spinning tyre. There has been less focus on the air related mechanisms than on other types of tyre noise generation mechanisms. This paper attempts to add some detail to current understanding of the air-related noise generation at the tyre road interface and gives some further information on how to identify the differences due to these mechanisms. Specifically in the present paper, a solid rubber tyre running on a vehicle chassis dynamometer is used to study the first two mechanisms. This is done with emphasis on the time history of the recorded signal and not on the frequency spectrum, as is more commonly used. A comparison with existing theoretical models of these mechanisms reveals some of the strength and weaknesses of the current understanding of these phenomena.

Keywords: tyre; road; noise; air pumping

\section{Introduction}

It is well known that road traffic noise is one of the major environmental problems in industrial countries. Because of significant 
reductions in powertrain noise, the main noise generation left from a passenger car is the one initiated by the tyre rolling over the road [14].

Tyre/road noise is generally divided into generation mechanisms and amplification mechanisms. One can divide the generation mechanisms into aerodynamic and vibration related phenomena $[1,4]$. Furthermore, the aerodynamic noise at the tyre road interface can be subdivided into four different mechanisms. The first one is named 'air turbulence', this is produced by the air which is moved due to the spinning tyre. The second mechanism, which is called 'air pumping', was introduced by Hayden in general terms in 1971 [2]. This term is used to describe the air being squeezed out of a cavity or groove when the tread hits the road surface (at the leading edge) and being sucked in again when the tyre lifts of the road surface (at the trailing edge). Another well-known effect for grooved tyres is the groove resonance, which is being initiated by the road closing the groove at the contact patch and therefore forming acoustic pipes. When the tyre rotates further and the tread lifts off the road surface, another mechanism comes into play, the 'air resonant radiation'. Introduced by Nilsson [5] in 1979 , it is a resonance where the air rushing back into the tread forms a spring - damper - mass system with the air underneath the tread. In the research reported in this paper only air related phenomena due to cavities in a tyre are considered. Note that there are also parallel investigations with a slick tyre and cavities on the road, as shown by Hamet [6], for instance. 
More specifically, in this paper, the 'air pumping' and 'air resonant radiation' mechanisms are examined experimentally using a cavity in a solid rubber tyre. The next section of the paper describes the existing theoretical understanding of these mechanisms. In section three, the specially designed tyre/road noise generation rig is described and the measurement method outlined. The resulting measurements made with this rig are analysed in section four. 'Air pumping' and ' air resonant radiation' mechanisms are identified and experimental results are compared to the theoretical models presented in section two. Finally, section five summarises the main findings of the research.

\section{Existing models of air-related noise generation mechanisms}

The initial air pumping mechanism introduced by Hayden [2] in 1971 is based on the formula of an acoustic monopole, where the generated pressure $p$ can be predicted by:

$$
p=\frac{\rho \cdot \ddot{V}}{4 \pi \cdot r} .
$$

Here $\rho$ is the mass density of the air, $\ddot{V}$ is the second time derivative of the volume displaced out of the cavity. The notation $r$ is used for the distance of the measurement point to the contact patch. Hayden [2] assumes that at the leading and trailing edges, due to the air 
movements, as shown in Fig. 1 (a), a monopole-like sound field is generated. He then modifies equation (1) by incorporating the tyre tread periodicity to predict the sound pressure level at a given frequency of repetition.

A different approach regarding the air pumping mechanism was carried out by Gagen [7] in 1999. Gagen argues that Hayden's model is not suitable for the effect of air being pumped out at the leading edge of the tyre because the simplicity of the monopole model does not reflect the complex air squeezing process at the tyre/road interface. His assumption is that the air responds sluggishly to local volume changes, in a similar manner to a damped oscillator, while the monopole theory equates local air movements exactly with the volume changes of the system. According to Gagen, the kinetic energy of air expelled from a linearly squeezed groove is

$$
E=\frac{\rho \cdot w \cdot A^{3} \cdot l^{3} \cdot v^{2}}{2\left(1-\frac{A}{d_{0}}\right) d_{0}^{4}} .
$$

Where the vehicle forward speed is defined as $v$ and the groove parameters are width $l$, length in circumferential dimensions $d$ and depth $w$. The variable $A$ is defined by Gagen as the new length of the cavity when it is fully compressed.

The second air related noise phenomenon for a tyre rolling over a road is the Helmholtz resonance or 'air resonant radiation'. In 1979 Nilsson et al. [5] identified this mechanism and proposed a model to describe it mathematically. According to reference [5], this noise 
source can be the dominant one in specific cases. As illustrated in Fig. 1 (b), the air resonance radiation only occurs at the trailing edge of the tyre as it is initiated by the tread groove or cavity leaving the roads surface. The acoustic signal, which is generated by the resonance radiation, is generally described in the form of a decaying swept sine wave. When the cavity leaves the road, a high amplitude sound of medium frequency is emitted. As the cavity proceeds further away from the contact area, the frequency rises whilst the amplitude decays. The model that Nilsson developed is for the determination of the frequency content only and does not predict the amplitude of the sound. It is based on a simple mass, spring and damper system, where the air surrounding the cavity is seen as the mass and the volume of the cavity acts as the spring and damper. With these assumptions, Nilsson [5] derives the following expression to determine the frequency as a function of the position of the cavity in respect to the road surface:

$$
\frac{V_{0}}{s_{1} x_{1}}=\frac{1+\left(k x_{1}\right)^{2}}{\beta\left(k x_{1}\right)^{2} \cdot\left(1+\left(\frac{\gamma k x_{1}}{2 \beta}\right)^{2}\right)^{2}}-\frac{1-\frac{k x_{1}}{\tan \left(k x_{1}\right)}}{\left(k x_{1}\right)^{2}}
$$

In this equation, $k$ is the acoustic wavenumber. The rectangular area $S(x)$ of the air gap between the tyre and the road surface is illustrated in Fig. 1 (c). For the distance of the cavity from the trailing edge of the contact patch the variable $x_{1}$ is used. In equation (3), $s_{1}=s_{1}\left(x_{1}\right)=\left(x_{1}^{2} / 2 r\right) W$ is the area of the rectangle formed by the gap 
between the groove/cavity at a height $h=\left(x_{1}^{2} / 2 r\right)$ above the road surface and by the width of the tyre $W$, where $r$ is the tyre radius [5]. The volume of the cavity is noted as $V_{0}$. In addition, two further variables are introduced: $\beta$ and $\gamma$. The values for these are adjusted empirically to account for the sideways opening of the 'acoustic horn' between the tyre and the road surface and account for the mass and the resistive impedance respectively. The equation (3) is used to compute the changing frequency of the sound via the wavenumber, with respect to the distance $x_{1}$

\section{Experimental setup and measurement method}

Figure 2 shows a schematic picture of the experimental rig. Only a half of the chassis dynamometer (for one tyre) was used for the experiments. The other half was fully covered by a thick wooden plate $100 \times 100 \times 3 \mathrm{~cm}^{3}$ to minimize the overall noise generated by the driving mechanism of the dynamometer. In the centre of the rig, a metal frame is shown, which supported the roller that rested on one drum of the chassis dynamometer. The tyre's supporting structure was mounted on the left hand side only, with large bolts and rubber insulation. On the right hand side of the metal frame there was a possibility to attach weights to load the tyre. The frame design itself was inspired by a design presented by Graf et al. in reference [8]. 
To achieve a small tyre to drum ratio in order to approximate a flat road surface as closely as possible, very small wheels of $120 \mathrm{~mm}$ diameter were used to run on the $500 \mathrm{~mm}$ diameter chassis dynamometer drum. Each wheel consisted of a solid rubber tyre mounted on a rigid plastic rim. In this experiment two simple cavity models of tyre tread pattern were investigated. One tyre had a cylindrical cavity of $9 \mathrm{~mm}$ in diameter and $5 \mathrm{~mm}$ in depth cut into the rubber surface. In this paper this cavity is referred to as the 'large cavity'. The other tyre had a much smaller cylindrical cavity with a diameter of $2.5 \mathrm{~mm}$ and a depth of $2 \mathrm{~mm}$, this cavity is referred to as the 'small cavity'. Photographs of both tyres are shown in Fig. 3. The significant difference between cavity size in relation to the tyre size in both designs is shown clearly.

The whole setup was based around the chassis dynamometer, which is located in a room of dimensions $10 \times 10 \times 10 \mathrm{~m}^{3}$. However, the chamber did not have anechoic termination on the walls and ceiling. Therefore, acoustic shielding was introduced around the dynamometer motor. Furthermore, the dynamometer cooling system was switched off for the short period of the measurements in order to minimize the noise generated by the driving motor.

Two microphones, as indicated by the grey arrows in Fig. 2, were used, suspended by an isolated support frame (not shown) to record the leading and trailing edge signal simultaneously. They were positioned in the median plane very close $(40 \mathrm{~mm})$ to the edges of the tyre/road interface. This close proximity was chosen in order to record 
the maximum amount of tyre/road noise with a minimum amount of background motor noise. Measurements were made with different speeds of the dynamometer ranging from $5 \mathrm{~km} / \mathrm{h}$ to $41 \mathrm{~km} / \mathrm{h}$. Each recording lasted two seconds with the data for both channels being acquired by a multi - channel spectrum analyser running at a sampling rate of 96000 samples/second. Some additional details of the experimental setup and measurement method are also given in reference [9].

\section{Results and discussion}

\subsection{Time histories for the leading edge and trailing edge recordings}

Fig. 4 shows the leading and trailing edge time history signals of the tyre with the large cavity at a tyre speed of $41 \mathrm{~km} / \mathrm{h}$. In Fig. 4 (a) the leading edge recording is shown. It can be seen that very low amplitude noise obscures the actual tyre road sound. This noise can be attributed to a low frequency (sub $1000 \mathrm{~Hz}$ ) noise generated by the chassis dynamometer motor. To remove this noise a digital bandpass $2^{\text {nd }}$ order Butterworth filter with a frequency range of $960 \mathrm{~Hz}$ and $24000 \mathrm{~Hz}$, was applied to the original time histories. The resulting leading and trailing edge filtered signals are shown in Fig. 4(b) and 4(c), respectively. It can be seen in Fig. 4(b) that the noise in the signal is less in comparison to the original unfiltered signal shown in Fig. 
4(a). Fig. 4(c) shows the filtered time history of the trailing edge recording. These bandpass filtered signals were used for subsequent analysis of aerodynamic noise phenomena. It can be seen in Fig. 4(c) that, in comparison to the peak amplitudes of the leading edge signal shown in Fig. 4(b), the peak amplitudes of the trailing edge signal are significantly higher. These very sharp pulses of short duration with a maximum of approximately $40 \mathrm{~Pa}$ occur every 0.0334 seconds. This corresponds to the repetition frequency of $29.06 \mathrm{~Hz}$ due to the reoccurrence of the cavity lifting of the dynamometer drum when running at a speed of $41 \mathrm{~km} / \mathrm{h}$.

Figure 5(c) shows the frequency spectrum of the filtered signal of the trailing edge recording shown in Fig. 4(c). In addition, the filtered signals from other rotation speeds are shown: Fig. 5(a) shows the $19 \mathrm{~km} / \mathrm{h}$ signal; and in Fig. $5(\mathrm{~b})$ the $31 \mathrm{~km} / \mathrm{h}$ is shown. All spectra have been determined for the whole $2 \mathrm{sec}$. long signal and no window was applied. Due to the bandpass filtering the low frequency components of all three signals are reduced. However the remaining frequency content is very similar for all the three speeds. Note that there is a dominant broadband frequency region from approximately 2000 to $6500 \mathrm{~Hz}$. This can be attributed to the frequency characteristics of the single pulses shown in Fig. 4.

For the highest rotation speed the broadband frequency content also has the highest relative amplitude. The fine structure of the broadband frequency content is controlled by the repetition frequency of the cavity as it 'contacts' the road [9]. Thus the broadband 


\section{S.J.Walsh@lboro.ac.uk}

frequency content shown in Fig. 5 actually consists of sharp harmonics with a frequency spacing corresponding to the rotational speed of the wheel.

To discover potential air related noise generation mechanisms individual pulses of the leading and trailing edge signals were further investigated. Fig. 6 shows an expanded view of one pulse of the time histories seen in Fig. 4. Both the leading edge (solid line) and trailing edge (dashed line) signals are displayed. In Fig. 6(a), the signals recorded for a tyre speed of $19 \mathrm{~km} / \mathrm{h}$ are displayed. Fig. $6(\mathrm{~b})$ shows the pulse time history when the tyre is running at $31 \mathrm{~km} / \mathrm{h}$, and in Fig.5(c) the corresponding signals at $41 \mathrm{~km} / \mathrm{h}$ tyre speed are shown. The sharp peak of the leading edge signal (identified by the arrow) can be attributed to when the cavity first 'contacts' the drum surface. Immediately after this when the cavity is completely covered by the drum there is no sound. When the cavity eventually lifts of the drum at the trailing edge, a larger amplitude oscillating signal is recorded by the trailing edge microphone. This sound is also evident in the leading edge signal but with a slight phase shift and significantly lower amplitude. It can be seen that, due to the different rotation speeds, the duration from the leading edge pulse to the initial trailing edge sound becomes shorter with increasing rotation speed. It is also evident that the peak amplitudes of the leading and trailing edge signal increase with increasing speed.

It can be seen in Fig. 6 that there is a significant difference between the shape of the signals recorded at the leading and trailing 
edges. At the leading edge, a very sharp pulse of short duration is recorded, whereas on the trailing edge signals of significant oscillations with changing amplitude and frequency can be identified. Fig. 6(a) shows the lowest amplitude signals with a maximum value of about $1.5 \mathrm{~Pa}$ recorded at the leading edge and $6 \mathrm{~Pa}$ recorded at the trailing edge. For the tyre speed of $31 \mathrm{~km} / \mathrm{h}$ the maximum amplitudes are $5 \mathrm{~Pa}$ recorded at the leading edge and $20 \mathrm{~Pa}$ recorded at the trailing edge. Fig. 6(c) shows the highest amplitude signal with a maximum of about $9 \mathrm{~Pa}$ for the leading edge peak and about $37 \mathrm{~Pa}$ for the trailing edge oscillations.

\subsection{Analysis of the leading edge recordings}

To gain a better understanding of which aerodynamic noise generation mechanism can be linked to the different signals, both the leading and the trailing edge signals are analysed separately. Individual pulses from the leading edge recordings, made at the three different speeds, are plotted over time and corresponding distance in Fig. 7. The distance travelled by the rotating tyre is obtained by multiplying the time history by the tire speed. The dotted line shows the leading edge signal for $41 \mathrm{~km} / \mathrm{h}$, the dashed line shows the corresponding signal for $31 \mathrm{~km} / \mathrm{h}$, and the solid line is the corresponding signal for $19 \mathrm{~km} / \mathrm{h}$. As noticed earlier, the signal recorded at the fastest rotational speed also results in the highest amplitude pressure pulse. It can also be seen in Fig. 7(a) that the time 
duration of the pulse recorded at the three different speeds are very similar - at approximately $0.1 \mathrm{~ms}$. Fig. $7(\mathrm{~b})$ shows the three pressure pulses plotted against corresponding distance. Thus, the fastest speed produces the longest signal against distance.

Both histories, time and distance, are now analysed according to the following equation derived from the monopole theory underlying Hayden's model [2] and expressed by equation (1). Thus, a calculation of the air displaced from the original cavity volume can be made using

$$
\ddot{V}=\frac{\partial^{2} V}{\partial t^{2}}=\frac{p \cdot 2 \pi \cdot r}{\rho},
$$

and by integrating the recorded pressure signal twice with respect to time. For the signals plotted over distance, the following equation can be used [10]:

$$
V^{\prime \prime}=\frac{\partial^{2} V}{\partial x^{2}}=\frac{p \cdot 2 \pi \cdot r}{\rho \cdot v^{2}} .
$$

Thus, the displaced volume is obtained by integrating the pressure signal twice with respect to distance. Also is should be noted that because of the microphone position just at the leading edge there is assumed to be a mirror source underneath the road surface. Therefore, the sound pressure of equation (1) must be reduced by the factor two, as stated in reference [10].

In Fig. 7(c) and (d) the results of the displaced volume calculation made using equations (4) and (5) are plotted, respectively. It can be seen in Fig. 7(c) and 7(d) that for the three different speeds 


\section{S.J.Walsh@lboro.ac.uk}

the calculated volume change is significantly different. This would indicate that Hayden's model is not appropriate for the leading edge signals of the tyre. This is not surprising as the signals for the three different speeds have the same duration, which is not in accordance with Hayden's results [2], where it is assumed that there is a relationship between the cavity length (the dimension in the circumferential direction of the tyre) and the rotational speed of the tyre. There appears to be no relationship between speed and cavity length in Fig. 7(a). For example, the duration of each pulse is about $0.1 \mathrm{~ms}$ and the time the cavity, of $9 \mathrm{~mm}$ diameter, needs to be covered completely by the road surface for the highest speed of $41 \mathrm{~km} / \mathrm{h}$ is $0.79 \mathrm{~ms}$. So the pressure peak is only generated at the very end of the squeezing process for the cavity. This process is starting earlier for higher and later for lower speeds respectively. However, there is a relationship of the peak itself and the speed of the tyre. It is found that the peak pressures are linked with $v^{2}$ factor between them.

The result of the calculation for the cavity volume change due to the tyre being compressed by the load is about $4.5 \times 10^{-9} \mathrm{~m}^{3}$ for the highest speed shown in Fig. 7(c) and Fig. 7(d). This change is very small in comparison to the actual volume of the cavity of $3.5 \times 10^{-7} \mathrm{~m}^{3}$. It represents a volume change of only 1.2 percent, which is much lower than the expected change of 5-10 percent following from the static deformation estimate. For the lower speeds, the indicated 
volume changes are even lower - approximately 0.5 percent for $31 \mathrm{~km} / \mathrm{h}$ and 0.3 percent for $19 \mathrm{~km} / \mathrm{h}$.

A comparison of the measured data can also be made with the kinetic energy approach suggested by Gagen [7] and expressed by equation (2). To relate the measured pressure, $p(t)$, at the leading edge to the kinetic energy of the expelled air consider the time integral of the 'pseudo-intensity' [11] to be given by

$$
I_{T}=\frac{1}{\rho \cdot c_{0}} \int_{0}^{t} p^{2} d t
$$

Thus, the total sound energy emitted at the leading edge is given by

$$
E=4 \pi \cdot r^{2} I_{T}
$$

For each of the three speeds shown in Fig. 7, Table 1 shows the measured peak pressure, half of the total energy of the source calculated using Equation (7) and the percentage of volume change implied by equation (2). In this case, half of the total energy of the source is used for a comparison with the kinetic energy predicted by the Gagen's model. It can be seen from Table 1 that the predicted cavity volume change is within expected limits and, thus, supports Gagen's assumptions of the noise generation process at the leading edge. However, the calculated volume change was expected to be constant as it is assumed to be independent of the rotation speed of the tyre. As noted earlier, there is a ' $\mathrm{v}^{2 \text { ' }}$ relationship between the peaks in the time histories in Fig. 7(a), which is also apparent in Gagens's model. However, inspection of equation (6) indicates, this ' $v^{2}$ ' 
relationship between the peak pressure and speed is transformed into $\mathrm{a}$ ' $\mathrm{v}$ ' dependence between energy and speed.

\subsection{Analysis of trailing edge recordings}

As shown in Fig. 6, the actual duration of the three trailing edge signals are similar irrespective of the different speeds. Each signal at the trailing edge is dominated by the characteristics of a decaying swept sine or tone-burst signal. Therefore, one would expect that the 'air resonant radiation' model from Nilsson et al. [5] could be appropriate. Fig. 8 shows a graph of the changing frequency of oscillations of the trailing edge signal versus the distance of the centre of the tyre cavity from the edge of the contact patch as the tyre rotates. The solid line shows the predicted frequency calculated using equation (3) with the coefficients $\beta$ and $\gamma$ set to 0.13 and 0.3 , respectively, to obtain a best fit according to the method proposed by Nilsson et al. [5]. Thus, the 'air resonant radiation' model predicts that, as the cavity moves away from the area of contact, the frequency of oscillations increases from about $1000 \mathrm{~Hz}$ to a peak of $6300 \mathrm{~Hz}$ when the cavity is at a distance of $0.0125 \mathrm{~m}$ from the contact patch. Thereafter the frequency of oscillations decreases with increasing distance. The corresponding measured data is indicated in Fig. 8: the cross marks the data for the lower speed case of $19 \mathrm{~km} / \mathrm{h}$; the inclined cross is used for $31 \mathrm{~km} / \mathrm{h}$; and the double cross is for the $41 \mathrm{~km} / \mathrm{h}$ 
data. It can be seen in Fig. 8 that the agreement of the measured data to the model prediction is very good over the range $0.0025 \mathrm{~m}$ to $0.01 \mathrm{~m}$. Below $0.0025 \mathrm{~m}$, the agreement is not as good, this might be due to the fact that the cavity is still opening up at this stage.

Once the cavity is fully open, the measured data points exhibit better agreement with the model. At the very end of the process, beyond $0.01 \mathrm{~m}$, due to the noise the measured data shows less agreement with the model. This is probably due to the high level of noise on the, now decaying, frequency oscillations. It was shown in Fig. 5 that the amplitude of the trailing edge signal is greater at higher speeds. One can see that the amplitude of this signal is also proportional to $v^{2}$. Thus, in Fig. 8 it can be seen that the higher the speed, and hence higher amplitude signal is generated, the better is the fit of the measured data to the model at the end of the process when the cavity is further away.

Fig. 9 shows a comparison of Nilsson's model with the measured data from the tyre with the smaller cavity. The shape of the cylindrical cavity was similar, however, it had much smaller dimensions. The predicted results from Nilsson's model are shown as the solid line, and the measured values are displayed by the crosses of different forms. Due to the different cavity dimensions, the predicted frequency of oscillations now goes from $2500 \mathrm{~Hz}$ to a maximum of about $17000 \mathrm{~Hz}$ at a distance of $0.006 \mathrm{~m}$ away from the contact area. The measured values appear to fit quite well to the model, especially in the region from $0.001 \mathrm{~m}$ to $0.003 \mathrm{~m}$ where the frequency of 
oscillation is rising. At very close distances, below $0.001 \mathrm{~m}$, the fit between measured and predicted data is not the best, again probably due to the change of the cavity volume as it just lifts of the road. At far distances, beyond $0.003 \mathrm{~m}$, the measured data are again dominated by the chassis dynamometer noise, therefore, the results are not as satisfactory. At the highest speed of $41 \mathrm{~km} / \mathrm{h}$ the measurement data give the best agreement with the model. This again is because highest speed generates the highest amplitudes and is less influenced by the background noise of the chassis dynamometer.

\section{Conclusions}

A comprehensive experimental investigation of the air-related tyre noise generation mechanisms has been undertaken using small cylindrical cavities on solid rubber tyres. Time histories of generated acoustic pressure have been studied at leading and trailing edges of the cavities as functions of the vehicle speed and cavity dimensions. Comparison has been made of the obtained experimental results with the existing theoretical models associated with different noise generation mechanisms.

In particular, it has been shown that Hayden's model [2] of 'air pumping' can not be applied successfully to the leading edge signal as 
the pressure pulse duration was shown to be constant for different tyre speeds, which is not consistent with Hayden's model.

The approach of Gagen [7] seems to be more adequate since the values of the predicted cavity volume change are within plausible limits. However, it is difficult to find a consistent link between the measured energy values and the predicted energy of his suggested model. Indeed, because of the sharp pulse occurring at the leading edge, the process might be due to an 'impact' mechanism rather than to an actual air - related process.

When the cavity is lifted off the road at the trailing edge, the 'air resonant radiation' phenomenon is assumed to occur and, thus, the model proposed by Nilsson et al. [5] could be applied. Here the predicted results are in better agreement with the measured values for the two different cavities investigated. The coefficients $\beta$ and $\gamma$ of Nilsson's model needed to be adjusted empirically for the first tyre under test. Nevertheless, they were then kept constant for two different types of cavities.

Thus, it follows from the above that only one out of the existing three theoretical models, namely Nilsson's model [5] associated with the 'air resonant radiation' mechanism, can be applied to the tyres tested. There are no theoretical models that would describe adequately other air-related tyre noise generation mechanisms. Further research is required to develop such models, perhaps using numerical calculations to describe motion of air masses in and out of tyre cavities. 


\section{References}

[1] Sandberg U. Ejsmont JA. Tyre/road noise reference book. Kisa, INFORMEX, 2002.

[2] Hayden RE. Roadside noise from the interaction of a rolling tire with the road surface. Proceedings of the Purdue Noise Control Conference 1971: 59-64.

[3] Medical Research Coucil. The non-auditory effects of noise. Norwich, Report R10, Institute of Environment and Health, Page Bros, 1997.

[4] Kropp W. Larsson K. Wullens F. Andersson P. Tyre/road noise generation - modelling and understanding. Proceedings of the Institute of Acoustics 2004, CD-ROM.

[5] Nilsson N-A. Söderqvist S. Bennerhult O. Air resonant radiation - a possible mechanism for high frequency noise from crossbar tires. Stockholm, IFM Akustikbyran Research Report 6.084.02, 1979.

[6] Hamet J-F. Deffayet C. Pallas M-A. Air-pumping phenomena in road cavities. Proceedings of the International Tyre/Road noise Conference 1990: 19-29.

[7] Gagen MJ. Novel acoustic sources from squeezed cavities in car tires. Journal of the Acoustical Society of America 1999; 106: 794-801.

[8] Graf RAG. Kuo C-Y. Dowling AP. Graham WR. On the horn effect of a tyre/road interface, part 1: Experiment and computation. Journal of Sound and Vibration 2002; 259: 417431. 
[9] Eisenblaetter J. Walsh SJ. Krylov VV. Experimental investigations into the air pumping effect at the tyre/road interface.

Proceedings of the Institute of Acoustics 2006; 28(1): 356-361.

[10] Plotkin K. Fuller W. Monttroll M. Identification of tire noise generation mechanisms using a roadwheel facility. Proceedings of the International Tire Noise Conference 1979: 127-141.

[11] Fahy FJ. Elliott SJ. Acoustic intensity measurements of transient noise sources. Noise Control Engineering 1981; 17 (3): 120125. 


\begin{tabular}{llll}
\hline & $41 \mathrm{~km} / \mathrm{h}$ & $31 \mathrm{~km} / \mathrm{h}$ & $19 \mathrm{~km} / \mathrm{h}$ \\
\hline $\begin{array}{l}\text { Measured peak } \\
\text { pressure values }\end{array}$ & 8.019 & 3.929 & 1.341 \\
{$[\mathrm{~Pa}]$} & & $1.048^{\star} 10^{-8}$ & $1.643^{*} 10^{-9}$ \\
\hline $\begin{array}{l}\text { Calculated } \\
\text { energy values [J] }\end{array}$ & $4.538 * 10^{-8}$ & 7.8 & 5.8 \\
\hline $\begin{array}{l}\text { Gagen model [6] } \\
\text { predicted volume } \\
\text { change [\%] }\end{array}$ & 10.4 & & \\
\hline
\end{tabular}

Table 1 Measured peak pressure, calculated energy and predicted volume change at the three rotation speeds 

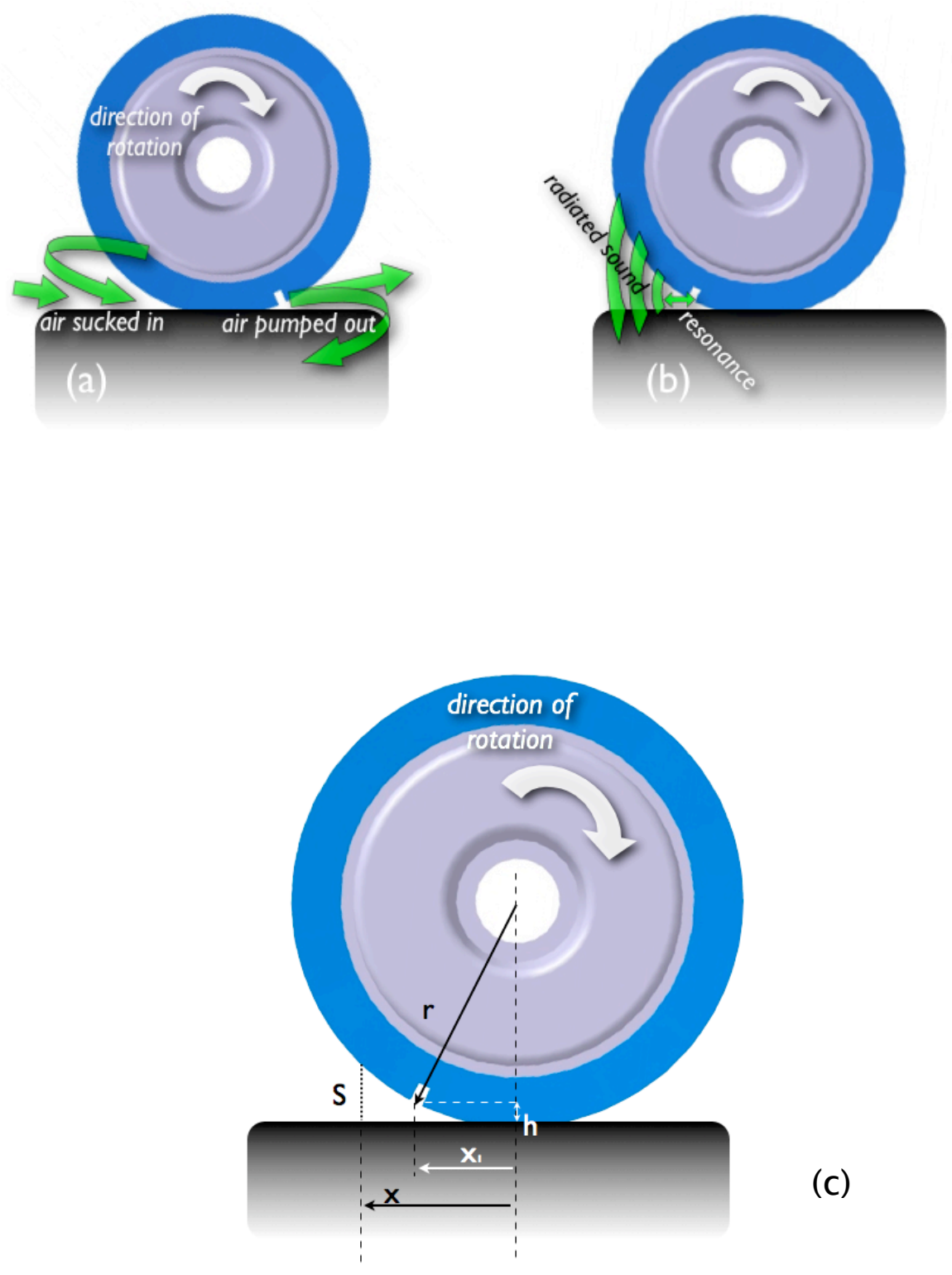

(c)

Fig. 1 Schematic illustration of the air-related effects:

(a) air pumping mechanism: air is pumped out at the leading edge (right hand side of the tyre) and sucked in at the trailing edge (left hand side of the tyre) as the tyre groove passes the contact patch; (b) air resonant radiation: occurs at the trailing edge of the tyre; (c) on the explanation of the variables in the equation (3). 


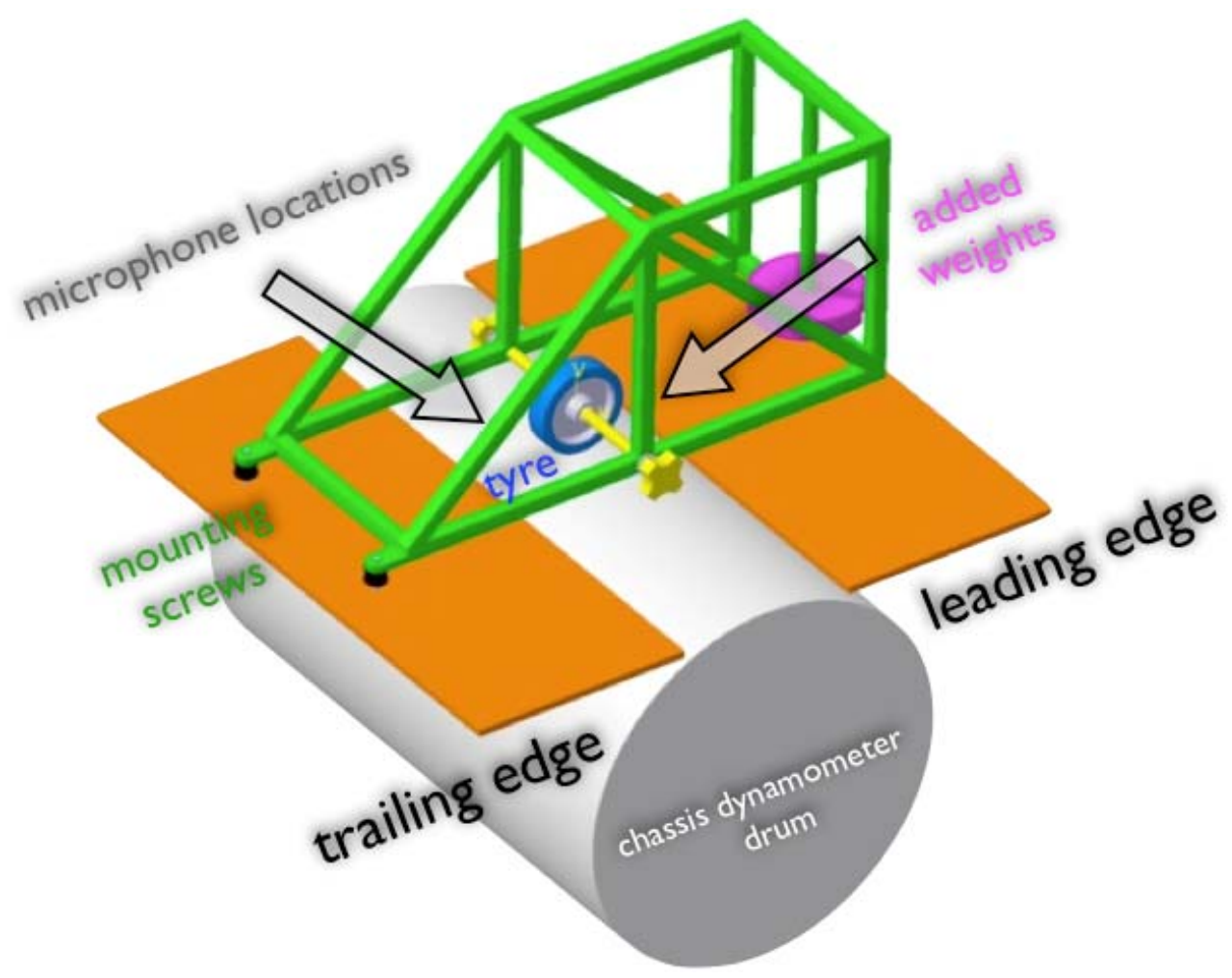

Fig. 2 Tyre mounted onto the chassis dynamometer with the two microphones (grey arrows) facing the leading and the trailing edge. 
a)

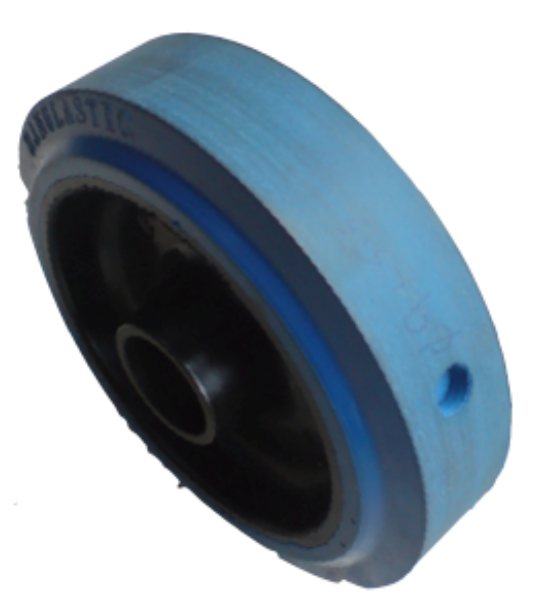

b)

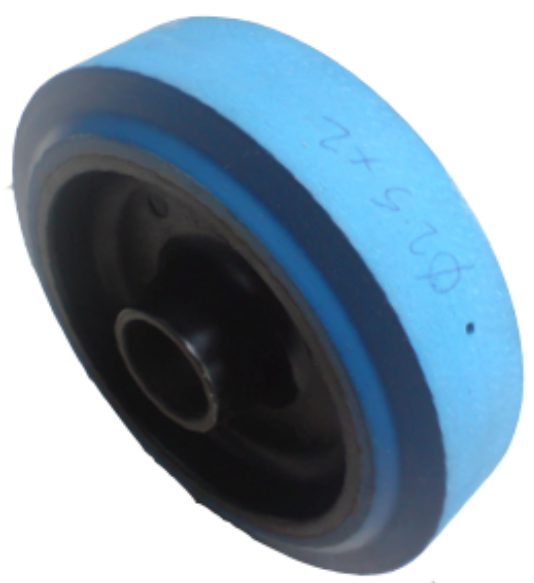

Fig. 3 Photograph of the two tyres used during the measurements: large hole (a), small hole (b) 
(a)

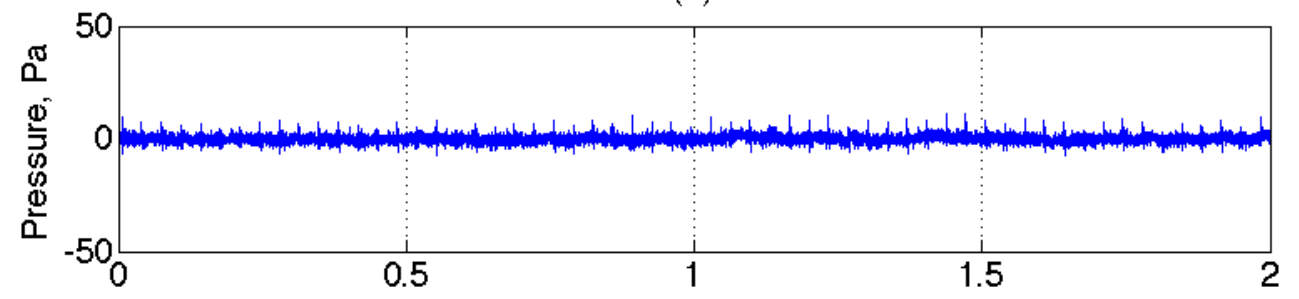

(b)

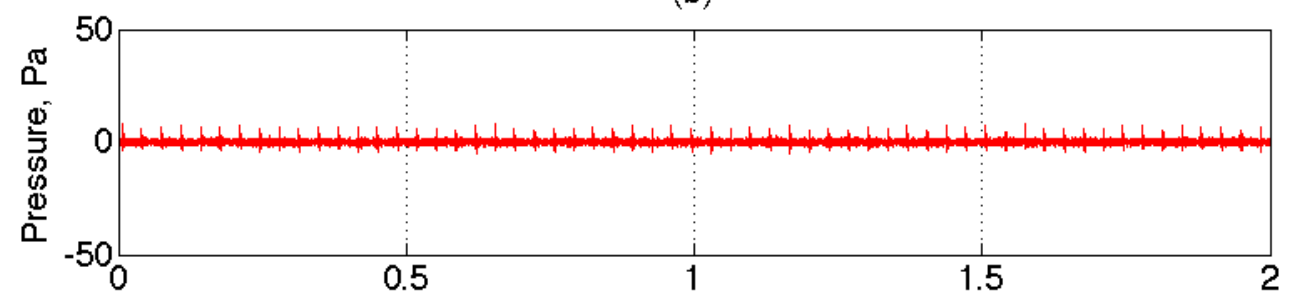

(c)

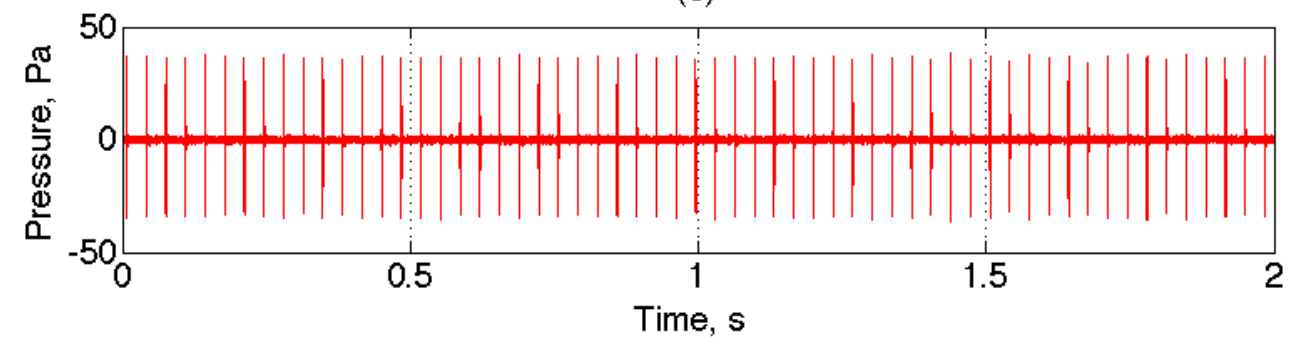

Fig. 4 Tyre/road noise due to the cylindrical cavity: (a) leading edge original time history; (b) leading edge filtered signal; and (c) trailing edge filtered signal. 
(a)

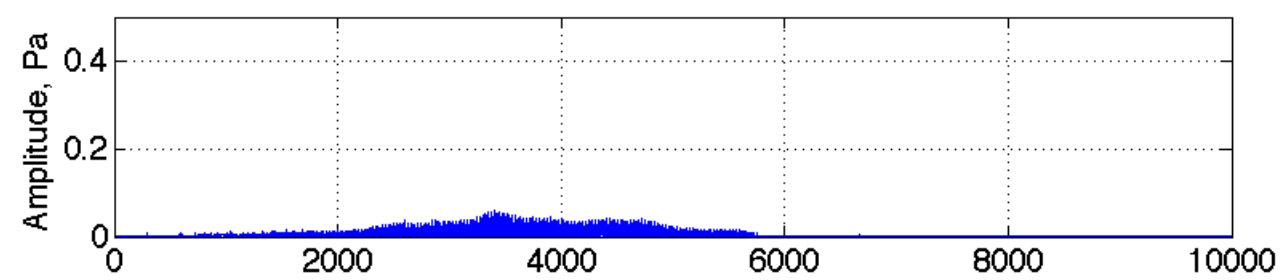

(b)

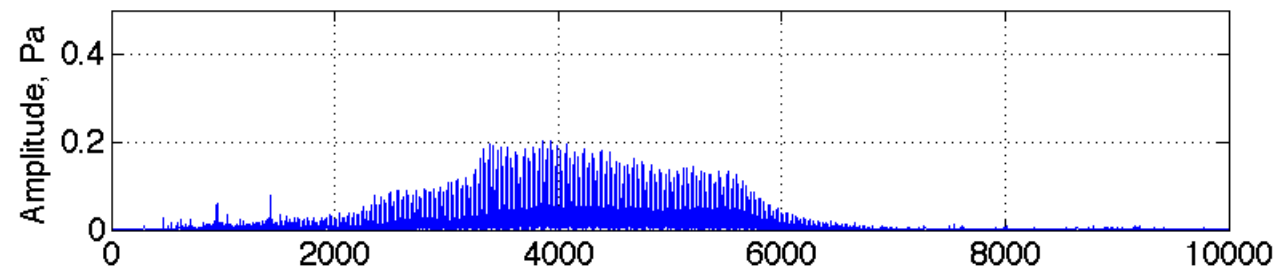

(c)

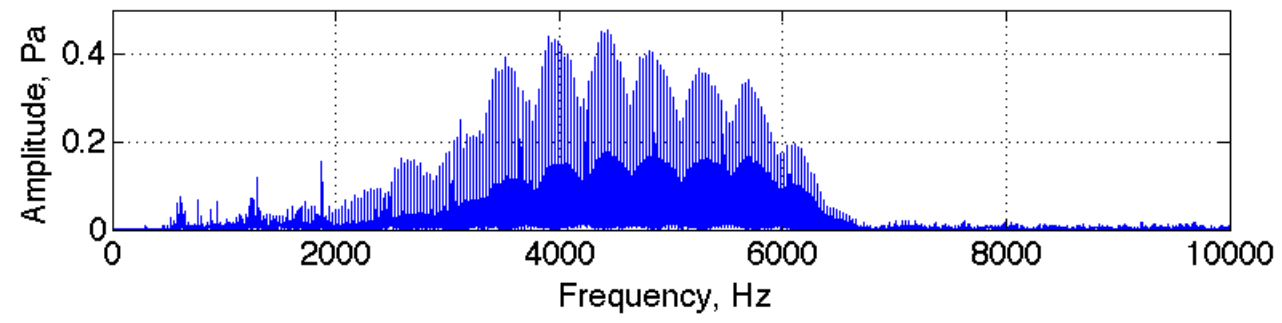

Fig. 5 Frequency spectrum of trailing edge signal, at different rotation speeds: (a) $19 \mathrm{~km} / \mathrm{h}$; (b) $31 \mathrm{~km} / \mathrm{h}$; and (c) $41 \mathrm{~km} / \mathrm{h}$. 
(a)

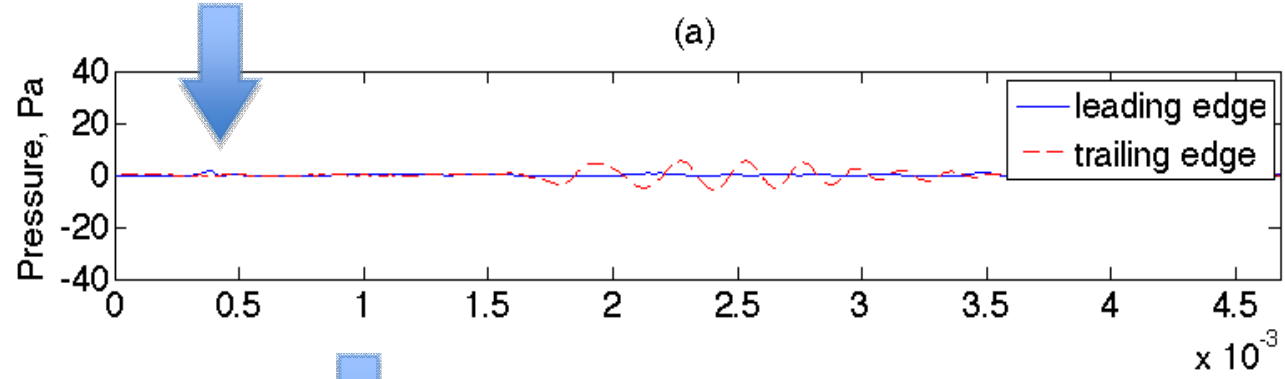

(b)

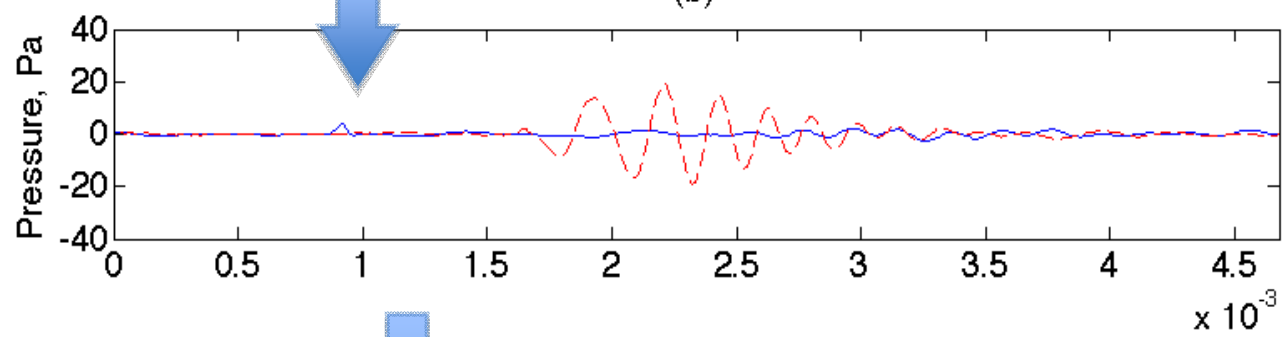

(c)

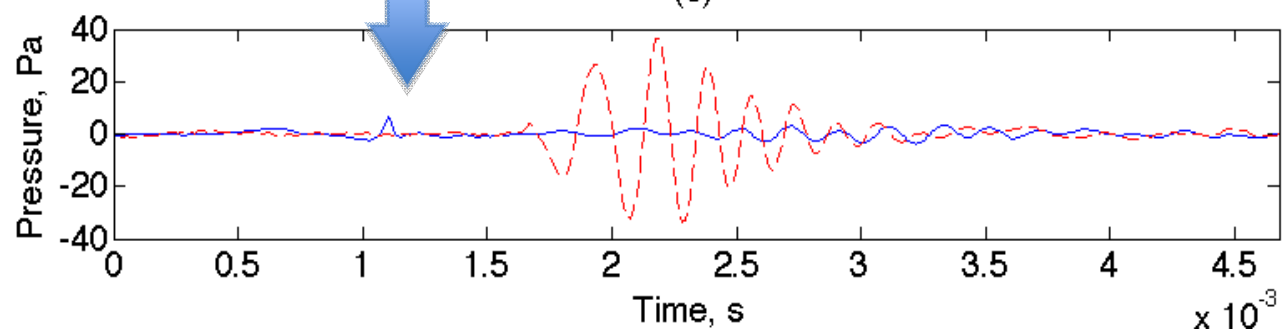

Fig. 6 Time histories of the leading edge (solid line) and trailing edge (dashed line) signals due to the contact patch event of the tyre with the large cavity at three different speeds: (a) $19 \mathrm{~km} / \mathrm{h}$, (b) $31 \mathrm{~km} / \mathrm{h}$ and (c) $41 \mathrm{~km} / \mathrm{h}$ 
(a)
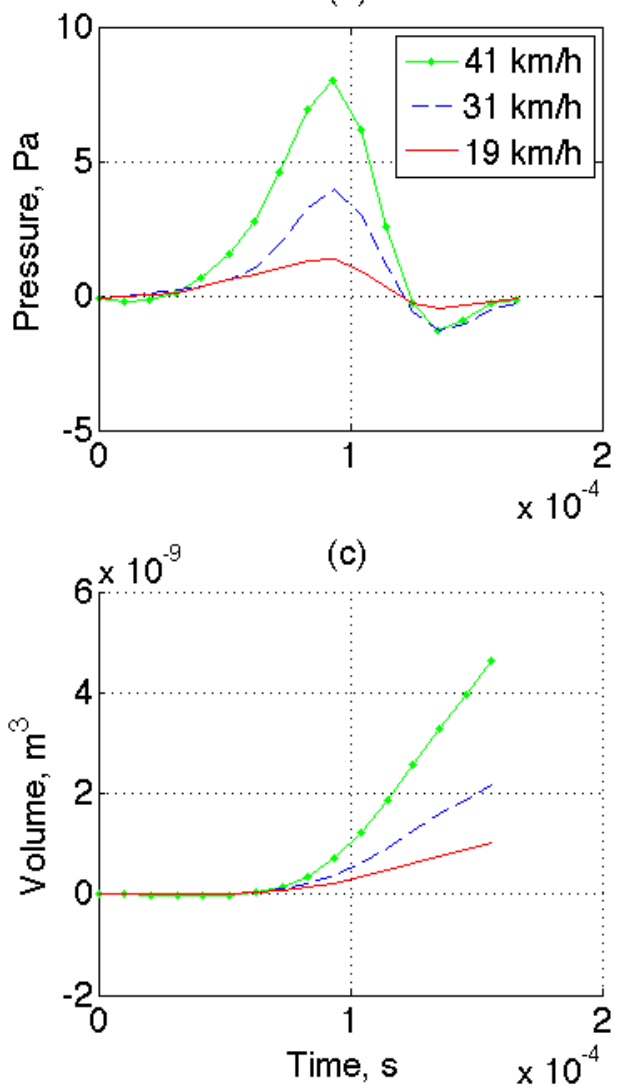

(b)

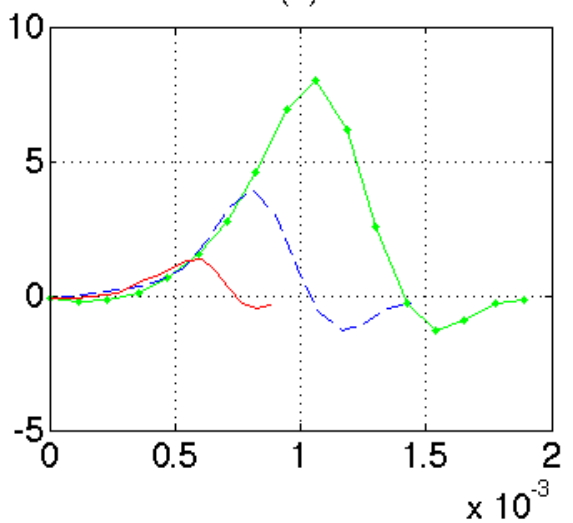

(d)

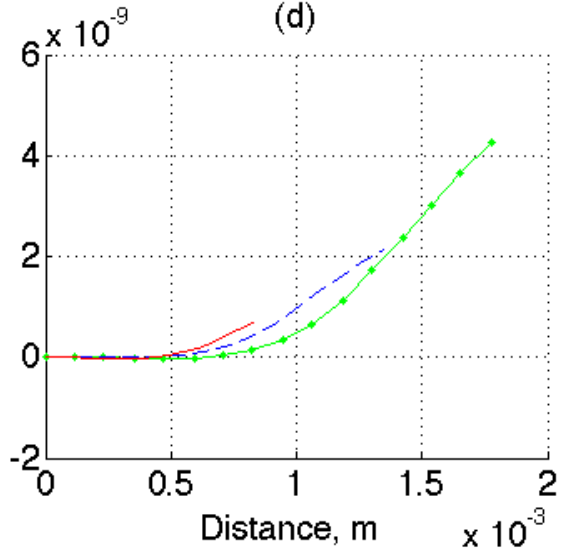

Fig. 7 Sound pressure pulses recorded at the leading edge at the three different speeds over (a) time and (b) distance; and prediction of the displaced cavity volume over (c) time and (d) distance. 


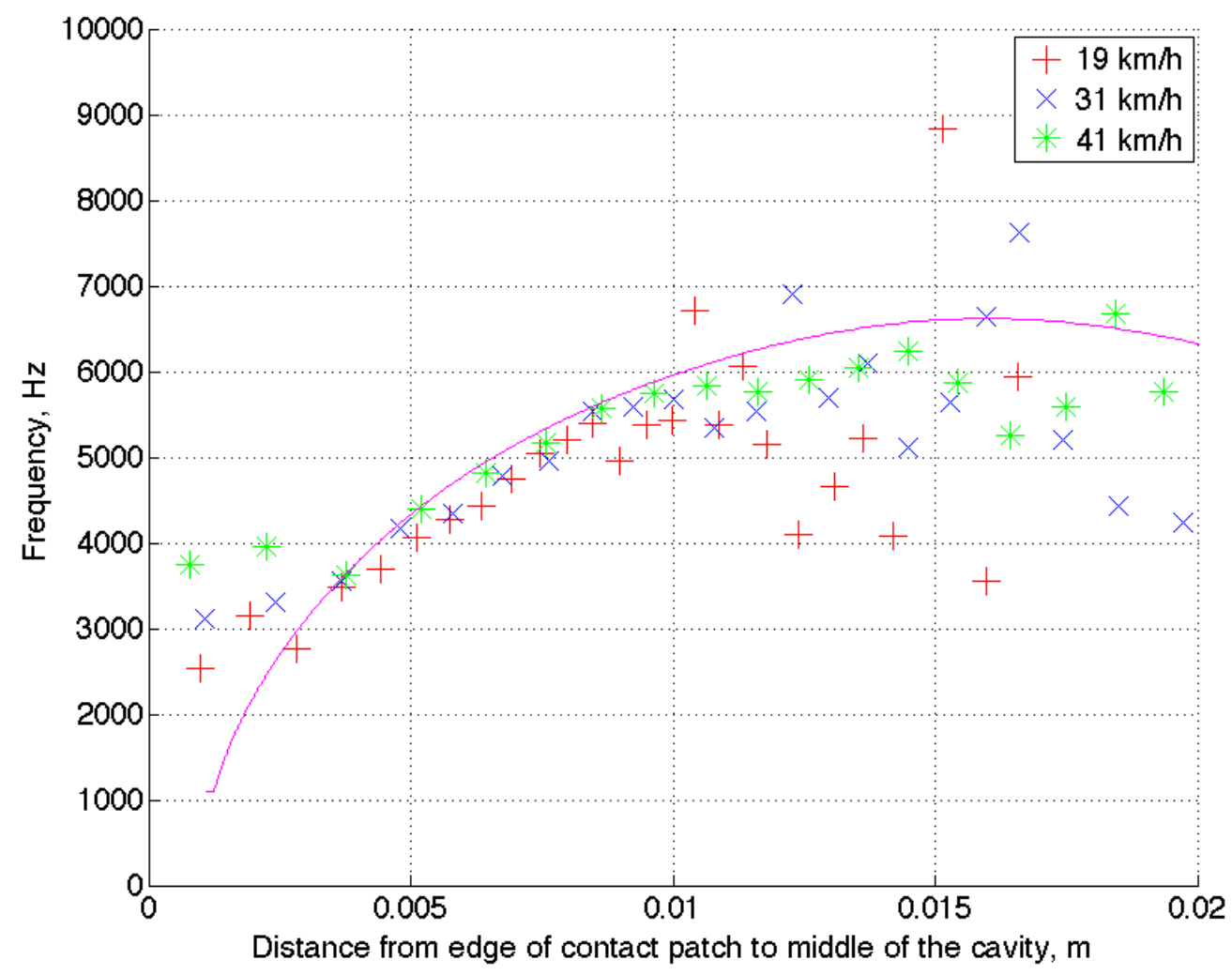

Fig. 8 Frequency of oscillation of trailing edge signals versus distance of the large cavity from the contact patch: (i) frequency measured from period of oscillations (crosses); (ii) predicted frequency assuming ' air resonant radiation' (solid line). 


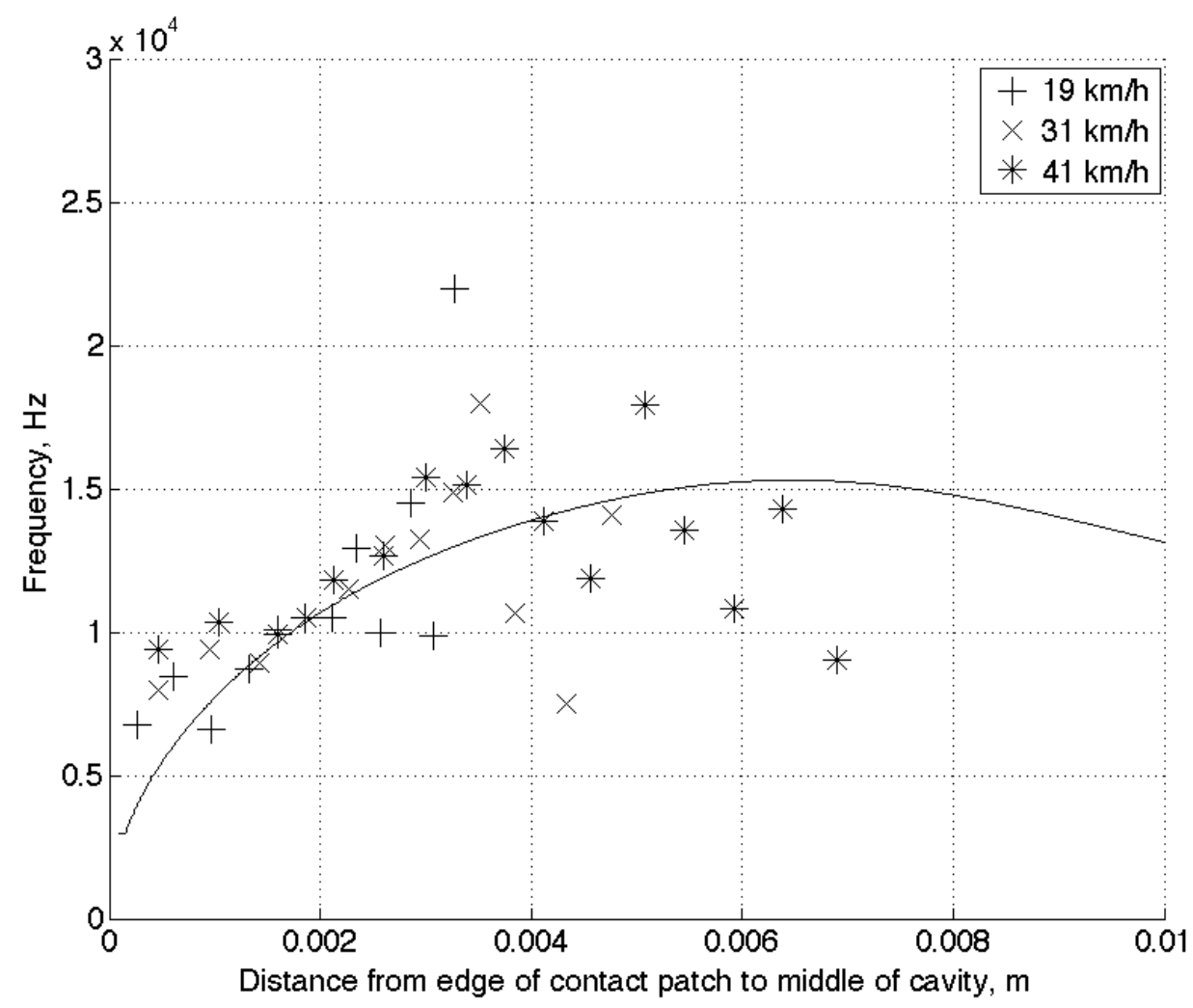

Fig. 9 Frequency of oscillation of trailing edge signal versus distance of small cavity from contact patch: (i) frequency measured from period of oscillations (crosses); (ii) predicted frequency assuming ' air resonant radiation' (solid line). 